\title{
Using intracellular plasmonics to characterize nanomorphology in human cells
}

\author{
Ahmad Sohrabi Kashani ${ }^{1}$, Alisa Piekny ${ }^{2}$ and Muthukumaran Packirisamy $\mathbb{1}^{1}$
}

\begin{abstract}
Determining the characteristics and localization of nanoparticles inside cells is crucial for nanomedicine design for cancer therapy. Hyperspectral imaging is a fast, straightforward, reliable, and accurate method to study the interactions of nanoparticles and intracellular components. With a hyperspectral image, we could collect spectral information consisting of thousands of pixels in a short time. Using hyperspectral images, in this work, we developed a label-free technique to detect nanoparticles in different regions of the cell. This technique is based on plasmonic shifts taking place during the interaction of nanoparticles with the surrounding medium. The unique optical properties of gold nanoparticles, localized surface plasmon resonance bands, are influenced by their microenvironment. The LSPR properties of nanoparticles, hence, could provide information on regions in which nanoparticles are distributed. To examine the potential of this technique for intracellular detection, we used three different types of gold nanoparticles: nanospheres, nanostars and Swarna Bhasma (SB), an Indian Ayurvedic/Sidha medicine, in A549 (human non-small cell lung cancer) and HepG2 (human hepatocellular carcinoma) cells. All three types of particles exhibited broader and longer bands once they were inside cells; however, their plasmonic shifts could change depending on the size and morphology of particles. This technique, along with dark-field images, revealed the uniform distribution of nanospheres in cells and could provide more accurate information on their intracellular microenvironment compared to the other particles. The region-dependent optical responses of nanoparticles in cells highlight the potential application of this technique for subcellular diagnosis when particles with proper size and morphology are chosen to reflect the microenvironment effects properly.
\end{abstract}

\section{Introduction}

Nanoparticles (NPs) with different physicochemical properties are being developed for a wide range of biomedical applications. Due to their small size and tunability, they are being explored for use in cancer diagnostics and treatment. NPs can be used as carriers to deliver drugs with greater efficiency than conventional methods. For example, with an NP delivery system, the release of drugs can be optimized under controllable conditions. The lack of selectivity and negative effects on healthy tissues/cells are the main drawbacks of classical

\footnotetext{
Correspondence: Muthukumaran Packirisamy (pmuthu@alcor.concordia.ca) 'Optical Bio-Microsystem Lab, Micro-Nano-Bio-Integration Center, Department of Mechanical, Industrial and Aerospace Engineering, Concordia University, 1455 De Maisonneuve Blvd. W., Montreal, QC H3G 1M8, Canada ${ }^{2}$ Department of Biology, Concordia University, 7141 Sherbrooke Street W., Montreal, QC H4B 1R6, Canada
}

methods ${ }^{1-3}$. NPs enable an advanced approach to developing therapeutic techniques in which NPs with unique physicochemical properties, nanomedicines, are designed to transport drugs to a specific place in the body or even in the cells. Before exploring their clinical use, it is essential to thoroughly evaluate NPs in cells to understand their toxicity and distribution and how they impact the intracellular environment and vice versa ${ }^{4,5}$. Detection and intracellular localization are critical steps in the development of nanomedicines and are extremely challenging because of the sensitivity and resolution limits of the current techniques. To understand nanomedicinebased treatment efficiency, we need to advance our ability to visualize NP uptake and identify/characterize NPs at the subcellular level ${ }^{6-8}$.

Various microscopic and spectroscopic techniques have been developed for the subcellular detection and 
visualization of NPs. Scanning electron microscopy (SEM) and transmitting electron microscopy (TEM) have high resolution and permit the visualization of NPs at the subcellular level. For example, Shapero et al. ${ }^{9}$ used TEM to visualize silica particles in A549 cells and found that these NPs are localized in lysosomes and not in the nucleus. Despite the advantages of TEM, this technique is costly and requires complicated sample preparation that can impact cellular structures ${ }^{10}$. Sample preparation for SEM is simple; however, this technique can image only the surface of cells. Light microscopy can be used to visualize both NPs and their surrounding environment; however, only clusters of particles can be visualized ${ }^{5}$. Recently, Chen et al. ${ }^{11}$ developed a superresolution fluorescence imaging technique with a spatial resolution of $10 \mathrm{~nm}$, well below the light diffraction limit. They used this technique to detect silica nanoparticles in HeLa cells, which they visualized in lysosomes and mitochondria. However, this method has limitations because it requires the addition of fluorescent tags onto NPs, which is restricted by their functionalization and requires a system capable of superresolution imaging.

Raman spectroscopy (RS) and infrared absorption (IR) have also been used to detect and localize NPs at the subcellular level ${ }^{12}$. The low spatial resolution of the IR technique has limited the application of this technique for intracellular diagnosis. Raman spectroscopy is a powerful technique and can differentiate chemical structures in cells based on their unique vibrational modes. Once a laser illuminates a sample, the emissions are shifted by the vibrational properties of molecules due to inelastic scattering $^{10,13,14}$. Noble NPs such as gold can enhance the intensity of Raman spectra in the vicinity of molecules, enabling the technique called surface-enhanced Raman spectroscopy (SERS). With SERS effects, the localization/ distribution of NPs at the subcellular level can be determined by Raman scanning over the entire cell ${ }^{15}$. The difficulty in interpreting the SERS spectra, and the timeconsuming operation for Raman scanning, are two main limitations of Raman techniques ${ }^{16,17}$.

Recently, our group monitored the intracellular location of NPs using plasmon properties ${ }^{18}$. For this technique, hyperspectral images (HSIs) in combination with darkfield imaging (DK) are utilized to characterize different subcellular components and approximate the locations of NPs. Over the past few years, hyperspectral imaging techniques have received considerable attention for single-cell analysis ${ }^{19-23}$. Both spectral and spatial information on NPs can be simultaneously recorded with hyperspectral imaging, providing a novel and label-free platform for the intracellular characterization of NPs. The entire spectrum can be acquired at each point in the HSI image, and in contrast to the SERS method ${ }^{24,25}$, no complex interpretation is required. With the HSI technique, high-resolution spectral information consisting of thousands of pixels can be recorded in less than one hour. With this technique, the localized surface plasmon resonance (LSPR) of gold and silver can be studied at the nanoscale to estimate their location and monitor their microenvironment in cells. The LSPR properties of NPs depend on their physicochemical properties, such as size, morphology, functionalization, and the surrounding medium. Once metallic nanoparticles are in cells, they interact with intracellular components. Hence, the particles are surrounded by biological environments with specific dielectric constants, resulting in core-shell nanohybrids. The optical properties of these nanohybrids are changed compared to those of the core parts (metallic particles $)^{26-28}$. The hyperspectral technique could provide information on these changes and on their intracellular localization. NPs can be characterized in cells much more rapidly using this advanced label-free method instead of SERS, while NP internalization can be studied by measuring unique spectral signatures.

Here, in the current paper, we used hyperspectral imaging to study the interactions of three different morphologies of gold NPs: nanospheres, nanostars, and Swarna Bhasma (SB), an Indian Ayurvedic/Sidha medicine, in A549 (human non-small cell lung cancer) and HepG2 (human hepatocellular carcinoma) cells. SBs are large gold-derived particles prepared as Ayurvedic/Sidha medicine and are variable in different compositions and shapes ${ }^{29,30}$. Three different aspects were studied in this work: (1) nanoparticle characterization in cells, (2) hyperspectral techniques for intracellular sensing, and (3) how nanomorphology could affect intracellular sensing. As described earlier, the LSPR of gold particles is sensitive to the surrounding medium, and we measured changes in plasmonic shifts to estimate the intracellular location and characteristics of particles in cells (Fig. 1). In addition, with this system, we could confirm the presence of NPs in cells by considering their unique spectral signatures. Our findings showed that the distribution of each type of gold particle is different. A greater accumulation of nanospheres than of other types of particles was observed in cells, and the nanospheres exhibited a greater plasmonic shift with higher sensitivity to their neighboring medium. This study revealed that the intracellular plasmon depends on the size and morphology of particles. The proposed subcellular detection method will help enhance our understanding of nanobio-interaction and develop nanomedicines for therapeutic and diagnostic applications.

\section{Materials and methods \\ Synthesis of gold nanostars and nanospheres}

The Turkevich method ${ }^{31}$, the most commonly used technique for preparing spherical particles, was used to 


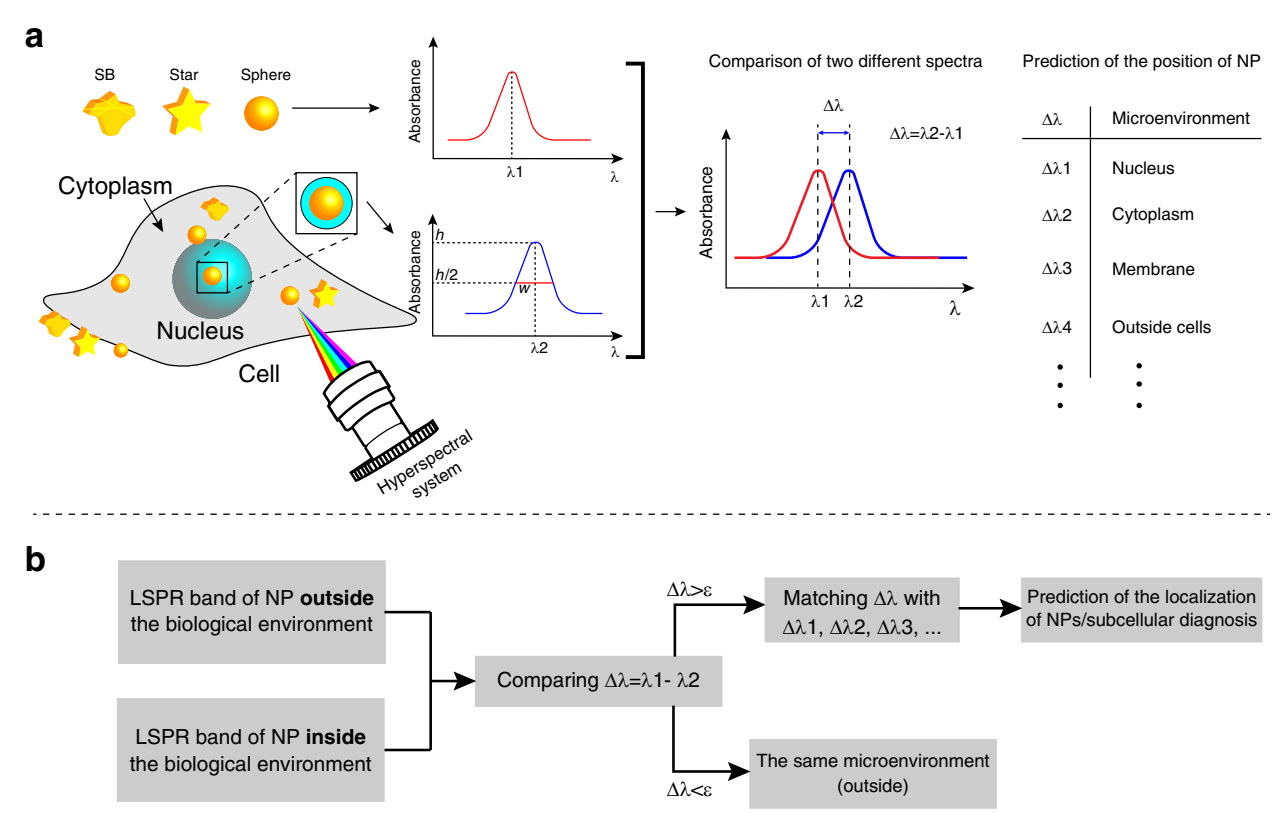

Fig. 1 Subcellular diagnosis using hyperspectral images and plasmonic shift. a A schematic showing how the detection/localization of NPS (with different morphologies) is determined within cells by the hyperspectral technique when their surrounding microenvironments are changed. b The concept of using hyperspectral images for subcellular detection/localization ( $\varepsilon$ shows very small differences in the LSPR band)

synthesize nanospheres by reducing gold chloroauric acid to gold atoms. Briefly, we boiled $80 \mathrm{~mL}$ of $40 \mu \mathrm{g} / \mathrm{mL}$ $\mathrm{HAuCl}_{4} \cdot 3 \mathrm{H}_{2} \mathrm{O}$ solution and then added $5 \mathrm{~mL}$ of $1 \%$ sodium citrate. We then heated the solution for $15 \mathrm{~min}$, and the solution changed from pale yellow to purple and then ruby-red. For the preparation of nanostars, a seedmediated approach was utilized. Ten milliliters of the $0.25 \mathrm{mM}$ gold solution was mixed with $10 \mu \mathrm{L}$ of $1 \mathrm{M} \mathrm{HCl}$ and $100 \mu \mathrm{L}$ of presynthesized seed solution. Ascorbic acid and silver nitrate were then simultaneously added to the solution and stirred for a few minutes. Then, the solution became blue.

\section{Swarna bhasma}

Swarna Bhasma (SB) particles are Indian gold-based particles that are prepared by the incineration of gold in the presence of herbal extracts. The incineration process is performed many times until their size reaches that of the chemically synthesized particles ${ }^{29,30}$. SB particles were purchased from Jaya Indian Medicine Pharmaceutical Pvt Ltd, Maduravoyal, Chennai, Tamilnadu, India in powder format, and they were suspended in deionized water.

\section{Hyperspectral imaging system and processing data}

A hyperspectral imaging system (CytoViva, Aruban, AL, USA) was used to capture HSI images of fixed NP-treated cancer cells. This system provides a high-resolution dark-field-based optical microscope combined with a photospectrometer for spectral characterization and spectral mapping at the nanoscale. The spectrophotometer is connected to a charged-coupled device (CCD) camera attached to a microscope to take images of the sample (Fig. 2). This method is a label-free technique and does not need any labels. This system permits capturing visible to near-infrared spectra in the range of $400-1000 \mathrm{~nm}$, with a resolution of $2.8 \mathrm{~nm}$ within each pixel of the scanned field of view. Each pixel of an HSI image is $\sim 25 \mathrm{~nm}$ using the $\times 100$ objective. Gold particles are brighter in HSI images, and they can be characterized by studying their spectral information. The LSPR properties of gold particles in the biological environment can be measured to identify and determine their spatial distributions in cells without damaging the sample ${ }^{32}$.

To capture HSI images, the condenser was adjusted until it touched the cover glass in the presence of the oil, and an oil-immersion objective $(\times 60$ or $\times 100)$ was then used to focus on the desired area. The exposure time for each spectral area was set to $0.15-0.25 \mathrm{~s}$, and the intensity was adjusted to a value between 1000-5000 units to ensure that spectral signals do not exceed the maximum limit. The desired area was scanned at $700 \times 700$ pixel resolution. We then used ENVI 4.8 (Exelis Visual Information Solutions, Boulder, CO, USA) software for processing the collected spectral data. We chose the desired regions of interest (ROIs), and the mean spectral data were collected for approximately 70-100 pixels. A spectral library (SPL) 


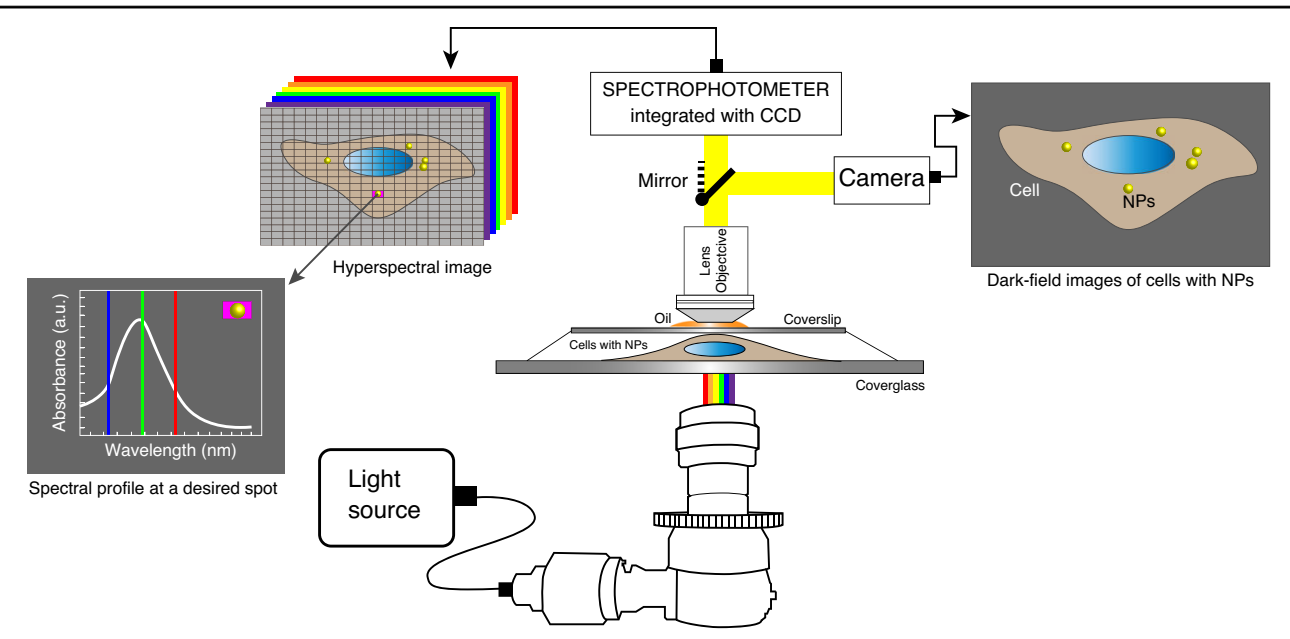

Fig. 2 Dark-field microscopy coupled to hyperspectral imaging to study gold nanoparticles in cells. A schematic showing the hyperspectral imaging instrument coupled with a dark-field imaging system to study the interaction of nanomaterials with cells.

was created from the ROI, and their spectra were filtered against the control cells (without particles) to obtain the spectral data on gold NPs (F-SPL). Furthermore, the filtered spectral data were calibrated with respect to the source light spectrum. Finally, the spectral intensities were normalized to between 0 and 1 to compare LSPR bands.

Spectral mapping was performed using the spectral angle mapper (SAM) algorithm to scan the HSI images and find pixels whose spectral profile is matched with the F-SPL with a threshold value of 0.1. In this algorithm, the intensity of the spectra does not affect the SAM analysis. The possibility of ignoring the intensity of the signal is an advantage of the SAM, as any variation in spectral intensity does not affect the results. A smaller difference shows a better match with the reference spectrum.

\section{Particle detection in different regions}

We used microscopic techniques to compare the plasmonic responses of particles in three different regions: inside the nucleus, at the periphery of the nucleus, and at the periphery of cells. We identified NPs that are very clearly inside the cells. We focused on the target particles/aggregates to obtain a high-resolution view of the particles, and we then changed the level of the focus to ensure that they are inside and not on the membrane or outside of the cells. Next, we used the ROI tool to find their average LSPR responses. We performed the same process for particles (with almost the same size) that were clearly inside the nucleus and on or outside the perimeter of cells to measure the plasmonic shifts. Note that once particles are aggregated, the dipole-dipole interactions between particles might affect their LSPR respons $^{33}$. To minimize the effects of dipole-dipole interactions on our comparison, we tried to compare aggregates with the same size in and out of cells. We applied the same strategy to quantify the uptake of particles in different regions of cells. Considering the size range of each type of particle (measured by DLS), we used ImageJ to count the average number of three different types of NPs in two different cell lines ${ }^{12}$. Student's $t$-test was used to compare statistical data, and differences were considered significant at $p<0.05$.

\section{Cell culture}

A549 (human non-small cell lung carcinoma) and HepG2 (human hepatocellular carcinoma) cells were used to characterize gold NPs in cells. A549 cells and HepG2 cells were cultured in F-12 medium and Dulbecco's modified Eagle's medium (DMEM; Wisent), respectively. Medium was supplemented with $10(\mathrm{v} / \mathrm{v})$ fetal bovine serum (FBS; Thermo Fisher Scientific), $2 \mathrm{mM}$ glutamine, $100 \mu \mathrm{g} / \mathrm{mL}$ streptomycin, and 100 units $/ \mathrm{mL}$ penicillin (Wisent). Cells were kept in an incubator at $37^{\circ} \mathrm{C}$ with $5 \%$ $\mathrm{CO}_{2}$ and passaged at $70 \%$ confluency.

\section{Cell fixation}

Cells were plated on acid-washed glass coverslips in 6-well dishes to $40 \%$ confluency, and then particles were added for $24-48 \mathrm{~h}$. Cells were fixed in fresh, ice-cold $10 \%$ w/v trichloracetic acid (TCA) and washed with TBST as previously described ${ }^{30}$ before placing the coverslip on a slide and sealing.

\section{Size and zeta potential measurements of particles}

We used a Zetasizer Nano ZS90 (Malvern Instruments Ltd., Worcestershire, UK) along with dynamic light scattering (DLS) analysis to measure the average size of all gold particles. The system is equipped with a red laser 
$(633 \mathrm{~nm})$ with a power of $4 \mathrm{~mW}$ and a detection angle of $90^{\circ}$. The hydrodynamic size of particles was measured in deionized water based on Brownian motion. Due to the different sizes of particles, light is scattered with different intensities. We added $1 \mathrm{~mL}$ of each suspension to a disposable (scratch-free) square cuvette. For each gold sample, we made ten DLS measurements and reported the average sizes in this study. Furthermore, we used a Zeta Plus electrophoresis instrument (Brookhaven Instrument Corp.) to determine the zeta potential of gold particles. The particles were introduced into a chamber containing two electrodes. An electric field was then applied to the electrodes. The particles travel with different velocities toward oppositely charged electrodes, depending on their surface charge. The migration of particles is illuminated with a laser beam, and the velocity is estimated based on the frequency shift in the scattered light. The velocity was measured and correlated to the zeta potential by the Henry equation $^{34}$. For our measurements, we set the temperature and $\mathrm{pH}$ at $25^{\circ} \mathrm{C}$ and 7.4 , respectively. For each sample, we analyzed the sample ten times, and the average was reported as the zeta potential of each type of particle.

\section{Results and discussion}

\section{Characterization of gold particles}

The physicochemical properties of three different types of gold particles were characterized by DLS, SEM, and potential zeta analyzers before using them in cells. DLS showed that the hydrodynamic diameter of the nanospheres is smaller than that of other nanoparticles, with an average size of $12 \mathrm{~nm}$ (Fig. 3a). Nanostars, as shown in Fig. 3b, tended to aggregate when dried on glass coverslips, and the size of the individual nanostars varied between 50 and $100 \mathrm{~nm}$ with an average size of $60 \mathrm{~nm}$. SB particles were significantly larger than nanostars and nanospheres. The crystal size of SBs was approximately $70 \mathrm{~nm}^{30}$, while the average size of the aggregates was up to $700 \mathrm{~nm}$ (Fig. 3c). With the use of electrophoretic light scattering, we measured the zeta potential of particles based on the Poisson-Boltzmann equation ${ }^{35}$. All particles displayed a negative potential; however, their levels were different. Synthesized particles showed negative zeta potentials due to the presence of citrate on their surface membrane. During the reduction process, both nanostars and nanospheres are decorated with citrates, which are the main source of the negative charge. Nanospheres showed a greater negative potential than nanostars, suggesting that they are more stable. Zeta potential measurements showed that the surface of SBs was coated with negative ions, but their zeta potential was less negative than that of the other particles. The elemental composition of SBs was reported in our previous studies ${ }^{29,30}$. Gold is the main element in SBs; however, they also contain other elements such as $\mathrm{Mg}, \mathrm{Ca}, \mathrm{Fe}$, and $\mathrm{Si}$.

\section{Hyperspectral imaging for particle characterization}

The particles were further characterized by hyperspectral imaging. Particles were dried on glass coverslips and sealed, and their LSPR spectra were measured. Depending on their size, the particles have different colors. The LSPR properties for the three different types of gold particles in this study are shown in Fig. 3d-f. SBs and nanostars are larger, with longer-wavelength LSPR bands. Nanospheres are smaller and more consistent in size, with LSPR bands of $560 \mathrm{~nm}$ for individual particles/small aggregates and between $650-750 \mathrm{~nm}$ for larger aggregates (Fig. 3d). Nanostars had two different peaks, one strong peak reflecting their spherical core and another weak peak corresponding to the branches (Fig. 3e). The band at $750 \mathrm{~nm}$ (particle \#1) belongs to branches, which become stronger when they get longer. The weaker signals confirm that branches are short, as shown in the SEM images. SB particles do not have any specific shape, and their LSPR bands are broader than those of the other particles. Smaller SBs have a band at $650-850 \mathrm{~nm}$, while larger SB particles have a broader band at $900-950 \mathrm{~nm}$, as expected for bulky gold materials (Fig. 3f).

\section{Enhanced dark-field images for the visualization of particles in cells}

Next, we characterized the particles in A549 and HepG2 cells. Cells were fixed after $24 \mathrm{~h}$ of treatment, and dark-field imaging was utilized to image the particles in cells with high resolution. Using this system, the noise-to-signal ratio is up to ten times lower than that of other optical instruments, providing enhanced nanoscale images of nanomaterials $^{36,37}$. In this system, the oblique angle of source illumination is adjusted such that it precisely focuses on the sample and bypasses the objective. This provides high contrast (very intense scatter from the sample and very dark background) to detect particles in cells.

Figure 4a, b shows DK images of cells with the different gold particles compared to nontreated cells. Due to the dark background, the gold particles are bright and easy to visualize. A549 and HepG2 cells had more nanospheres than nanostars or SBs. As shown in the DK images, nanospheres were localized mostly in the perinuclear regions. A549 cells appeared to contain more nanospheres than HepG2 cells. Nanostars were more randomly distributed in the cytosol in A549 and HepG2 cells, while cells had much fewer SBs. These larger particles may be more challenging for cells to take up ${ }^{30}$. Therefore, in contrast to nanospheres and nanostars, SB particles were not found in all cells, and they were relatively randomly distributed, similar to nanostars.

\section{Particle detection with hyperspectral methods}

As discussed earlier, HSIs can be used to detect particles such as gold NPs in cells. In Fig. 4c, d, typical HSI 
a
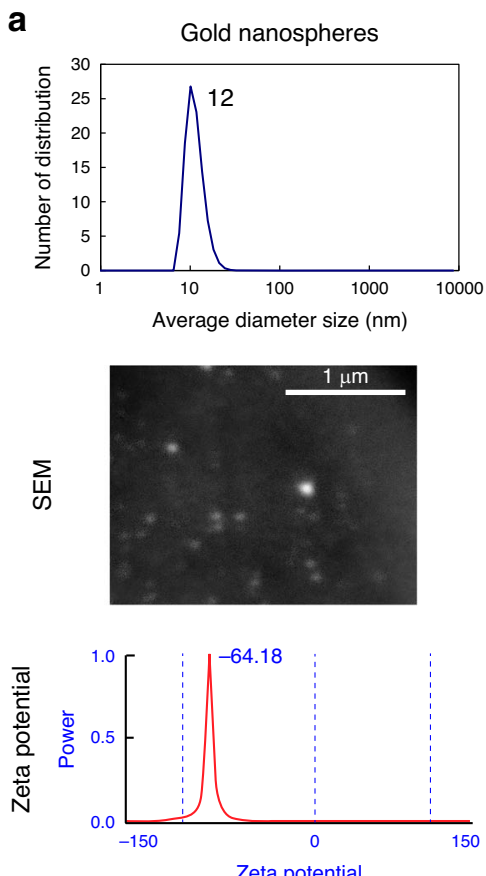

d
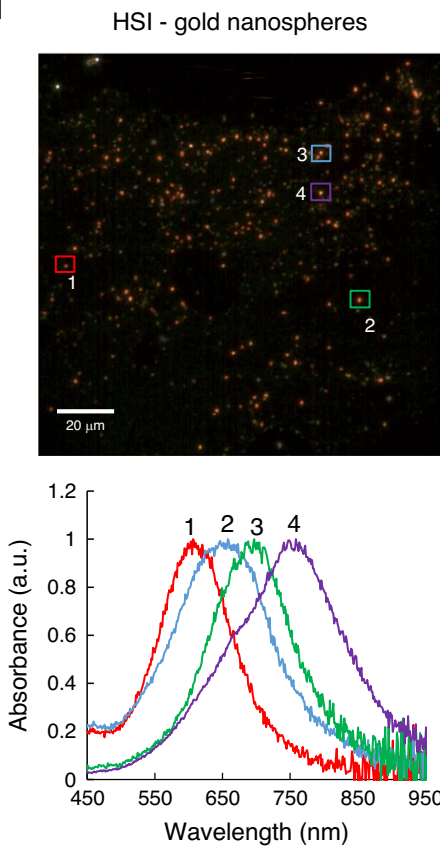

b
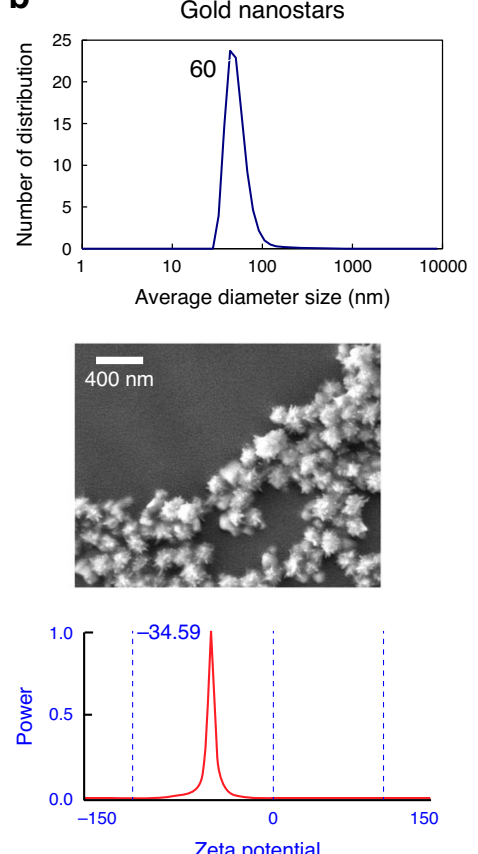

$\mathbf{e}$
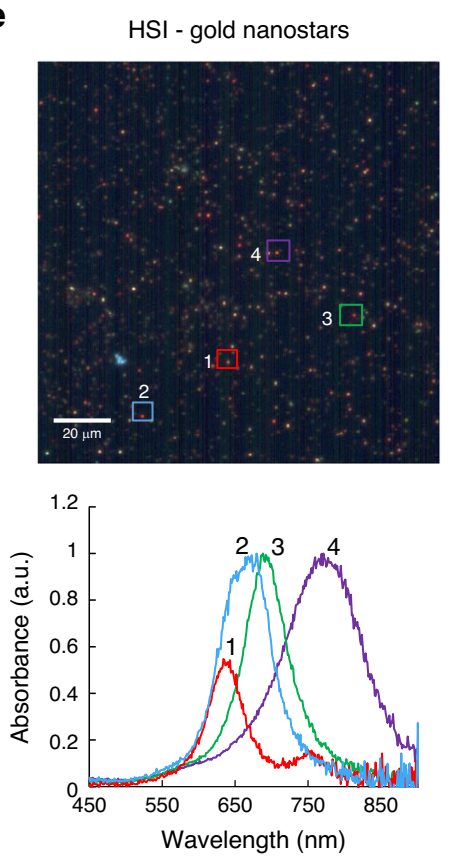

C
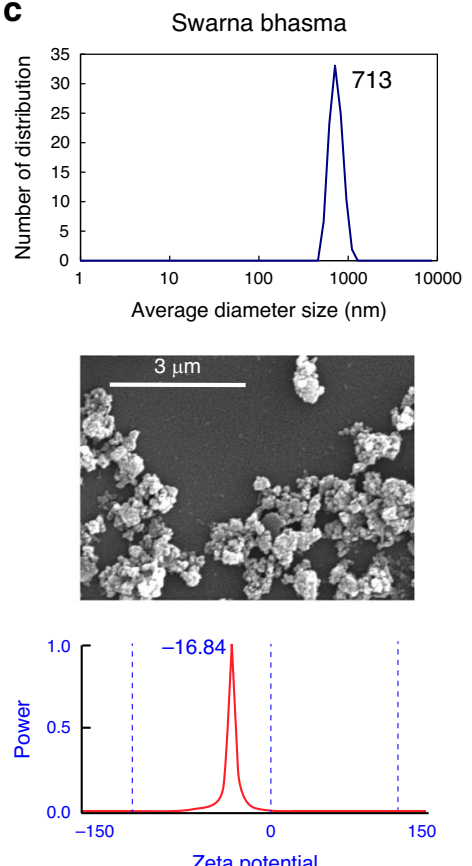

f $\mathrm{HSI}$ - swarna bhasma
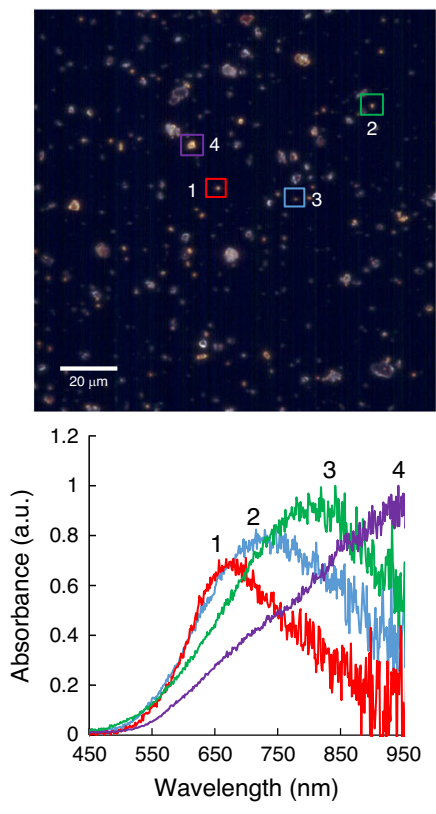

Fig. 3 Gold particles characterization using different techniques. a Average size of nanospheres measured by DLS, SEM images of nanospheres and their zeta potential. $\mathbf{b}$ The average size of nanostars (by DLS), SEM of nanostar aggregates, and their zeta potential. c Size distribution of SB particles suspended in deionized water (by DLS), SEM of SBs, and their zeta potential, $\mathbf{d}$ A hyperspectral image (top) of nanospheres and corresponding LSPR bands (bottom). e A hyperspectral image (top) of nanostars and corresponding LSPR bands (bottom). $\mathbf{f}$ A hyperspectral image (top) of SBs and corresponding LSPR bands (bottom)-for HSI image; scale bar is $20 \mu \mathrm{m}$

images of A549 and HepG2 cells are shown. Figure 4c, d show the absorbance spectra from different regions of the cell, including the cytoplasm (red) and nucleus (orange). The absorbance intensity of the nucleus was higher than that of the cytoplasm. The nucleus is more electron-dense than the cytosol and likely absorbs more 

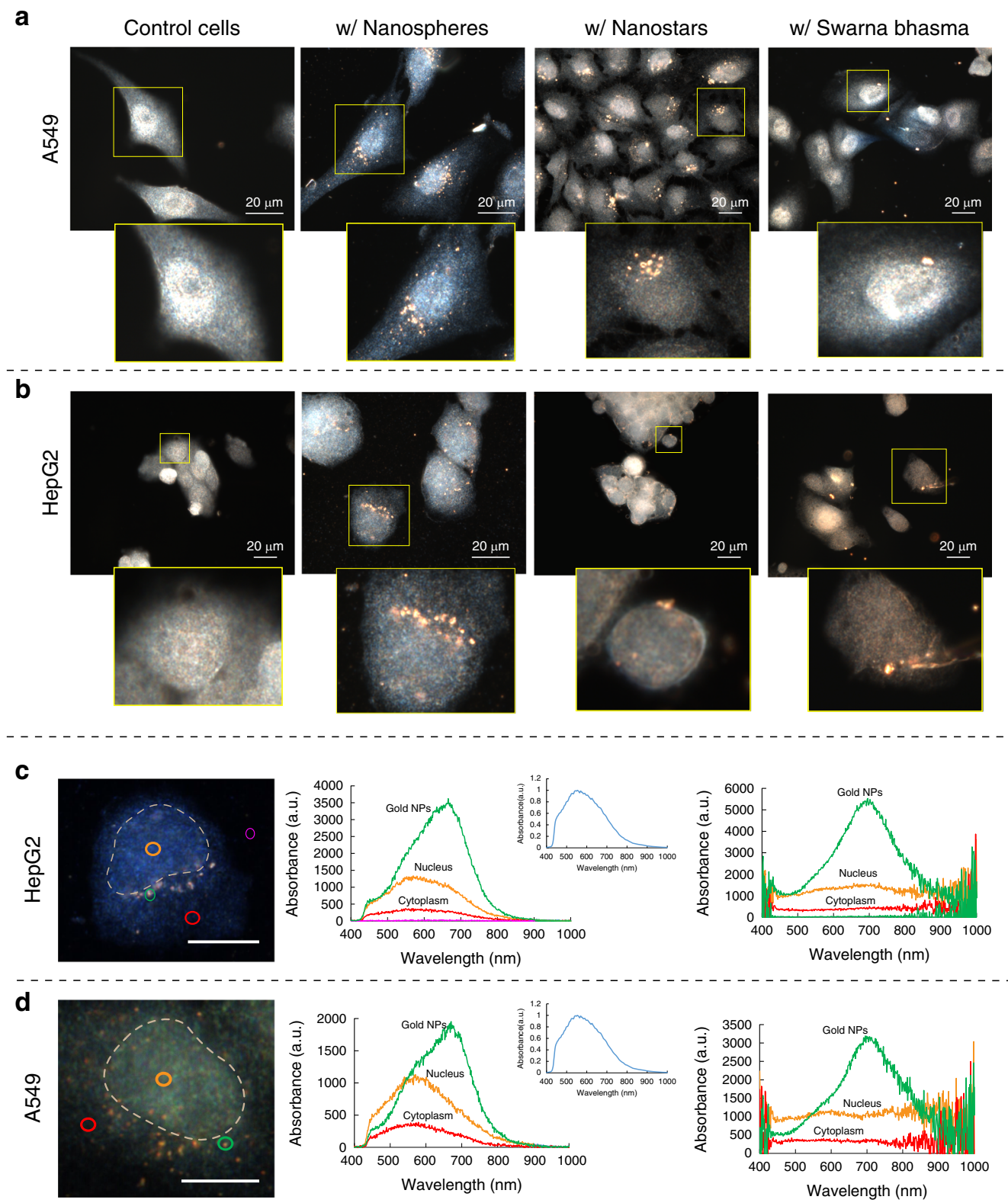

Fig. 4 Dark-field images of cells incubated with gold nanoparticles and their intracellular LSPR. a Dark-field images of A549 cancer cells treated with three different types of particles. b Dark-field images of HepG2 cancer cells treated with three different types of particles. Few SBs entered into cells, while nanospheres were found more often in cells. c Spectral responses from different regions of HepG2 cells (nucleus and cytoplasm), gold nanospheres in HepG2 cells, and corrected responses after normalization with respect to the halogen lamp spectrum (right panel). d Spectral responses from different regions of A549 cells (nucleus and cytoplasm), gold nanospheres in A549 cells, and corrected responses after normalization with respect to the halogen lamp spectrum (right panel). The scale bar is $20 \mu \mathrm{m}$

light. In Fig. 4c, d, the green profiles show the absorbance of the gold particle/aggregate in cells. Due to their specific LSPR properties, gold NPs display unique spectral information depending on their size and morphology, as well as their surrounding microenvironment. The band position of NPs in cells and how it changes can be used to detect them at the subcellular level. A large peak is seen between $600-700 \mathrm{~nm}$ corresponding to the gold nanoparticles. The cytoplasm and nucleus have peaks in the range of 500-600 nm, which comes from the light source (shown in Fig. 4c, d-inset). We normalized the HSI profiles to obtain more precise spectral responses. The normalized data are plotted in Fig. 4c, d (right panels). After correction, the spectra from the nucleus and cytoplasm are flat, and the gold particle/aggregate peak is at $700 \mathrm{~nm}$. The corrected spectral information 
allows the detection of particles in cells and characterizes their properties in cells.

\section{Particle mapping in hyperspectral images}

Different particles in cells might not be detected visually with HSI images. Scanning through the spectral data of an HSI image can be utilized to distinguish pixels that match our desired spectra. The spectral angle mapper algorithm was used to scan HSI images spectrally. Before performing SAM analysis, a spectral reference was defined for gold particles to classify the different pixels in the HSI images. With SAM, the spectral similarity is measured between two spectra. With this algorithm, each spectrum was assumed to be a vector (with a direction and length), and the angle between the vector at each pixel and the F-SPL vector (gold particles) was calculated and compared to the threshold. With the SAM algorithm, we could detect pixels whose bands are similar to gold NPs and diagnose particles that are not visible in HSI images. This technique can be used to determine the distribution/localization of particles within cells.

Due to the lack of resolution of HSI images compared to DK images, spectral measurements were used to detect the particles. Figure 5a shows the mapping of three different types of particles in A549 cells with respect to SPL shown on the right panel. With the SAM algorithm, the particles are labeled in red after mapping to show their distribution in cells. Nanospheres were enriched in perinuclear regions, although they could be seen in various regions of the cell, including the nucleus. Zooming into a single cell, the mapped image shows nanospheres in the nucleus, although they were not visible in the original HSI image. Nanostars were more aggregated and localized more randomly than nanospheres, with only a few in the nucleus. SB particles are larger, and the mapped image does not provide further information on their distribution in cells. As shown in the right panel, their spectra varied more, likely due to their irregular morphology. Similar mapping was performed on HepG2 cells, as shown in Fig. 5b. The distribution patterns of the three different types of gold particles in HepG2 cells were similar to those in A549 cells. HepG2 cells had more nanospheres and nanostars than SB particles. However, HepG2 cells appeared to have fewer nanospheres and nanostars than A549 cells. There was no significant change in SB distributions between the two cell lines, and only small aggregates were in cells, with larger particles on the membrane surface. We observed a few individual/aggregates of nanospheres in the HepG2 nucleus, and their level of internalization was less than that of A549 cells.

\section{NP spectral responses for intracellular diagnosis}

Plasmon particles can also be used for intracellular sensing and the imaging of biomarkers in cells. Different strategies have been employed to take advantage of the optical properties of plasmon particles to detect/visualize subcellular components and biomarkers in cells. For example, Kumar et al. ${ }^{38}$ utilized functionalized gold NPs to bind actin biomarkers in cells to provide strong molecular-specific optical signals. As mentioned earlier, plasmon particles can also enhance Raman signals to provide SERS probes for intracellular detection and imaging. All these techniques rely on optical enhancement because of the LSPR properties of NPs. In addition to optical enhancements, plasmon NPs can be used as sensing tools to detect biomarkers based on their LSPR changes. Plasmonic or LSPR shifts have been widely used to develop biosensors; however, to the best of our knowledge, this technique has not been used for intracellular diagnosis. The LSPR of gold NPs is influenced by size, morphology, and the local microenvironment. The LSPR scattering maximum wavelength is sensitive to the microenvironment and its optical properties, such as the dielectric constant or refractive index. Therefore, any change in the local environment of gold NPs can cause a shift in the LSPR band (plasmonic shift). This change can be quantified through the following equation ${ }^{39,40}$ :

$$
\Delta \mathcal{U}_{\max }=m \Delta n\left[1-\exp \left(\frac{-2 d}{l_{d}}\right)\right]
$$

where $\Delta \lambda_{\max }$ is the maximum expected plasmonic shift, $\mathrm{m}$ is the bulk refractive index of the NPs, $\Delta n$ is the refractive index change induced by the absorbance, $d$ is the thickness of the dielectric layer, and $l_{d}$ is the characteristic electromagnetic-field-decay length. This equation shows that the plasmonic shift is directly proportional to changes in the dielectric constant of the local environment. By increasing the dielectric layer thickness, the last term is increased, causing a higher shift in the LSPR band.

Shifts in the LSPR band of gold NPs in cells enable us to detect and sense small changes in the environment adjacent to particles and verify their presence in cells. Different regions in cells exhibit different microenvironments, which affect the optical responses of biomolecules or particles in their vicinity ${ }^{41}$. These effects are reflected in the LSPR responses. In Fig. 6a, b, and c, the LSPR bands of three different types of particles in HepG2 cells are illustrated. Gold particles were marked with different colors in different regions of cells (edge/outside of cells in red, cytosol in green), and their corresponding spectra were compared in the bottom panel. As shown in Fig. 6a, gold nanospheres outside cells or in the periphery of cells exhibited peaks at $\sim 693 \mathrm{~nm}$. Nanospheres inside cells had a shifted spectrum of $756 \mathrm{~nm}$. This relatively large shift is likely due to differences in the surrounding environment. This shift might also be related to other parameters, including their interactions with intracellular components, 
a
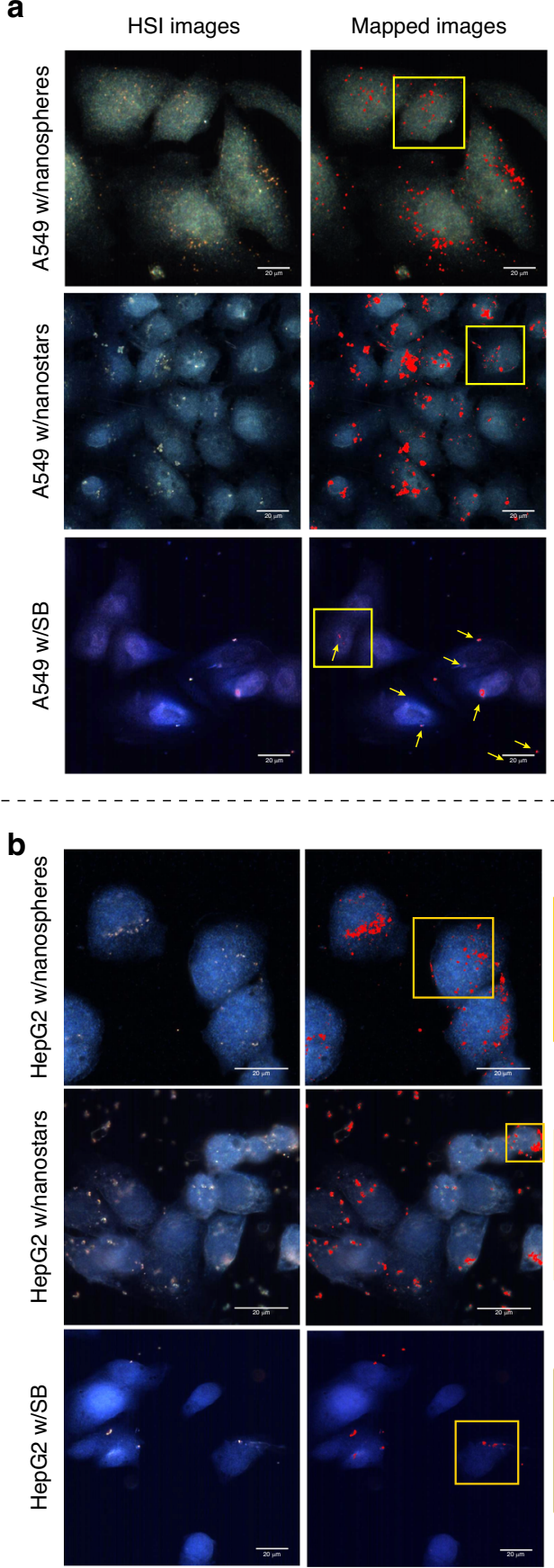
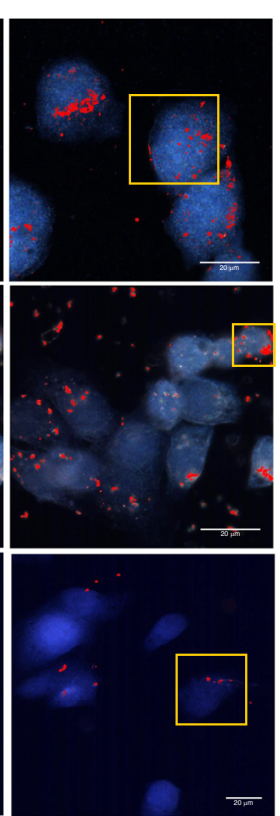

Mapped images
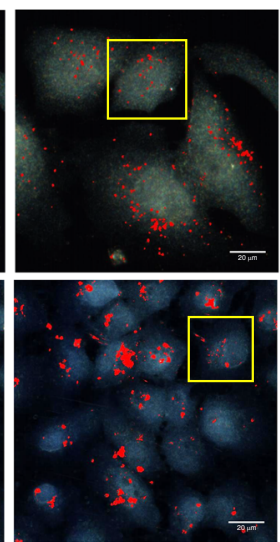
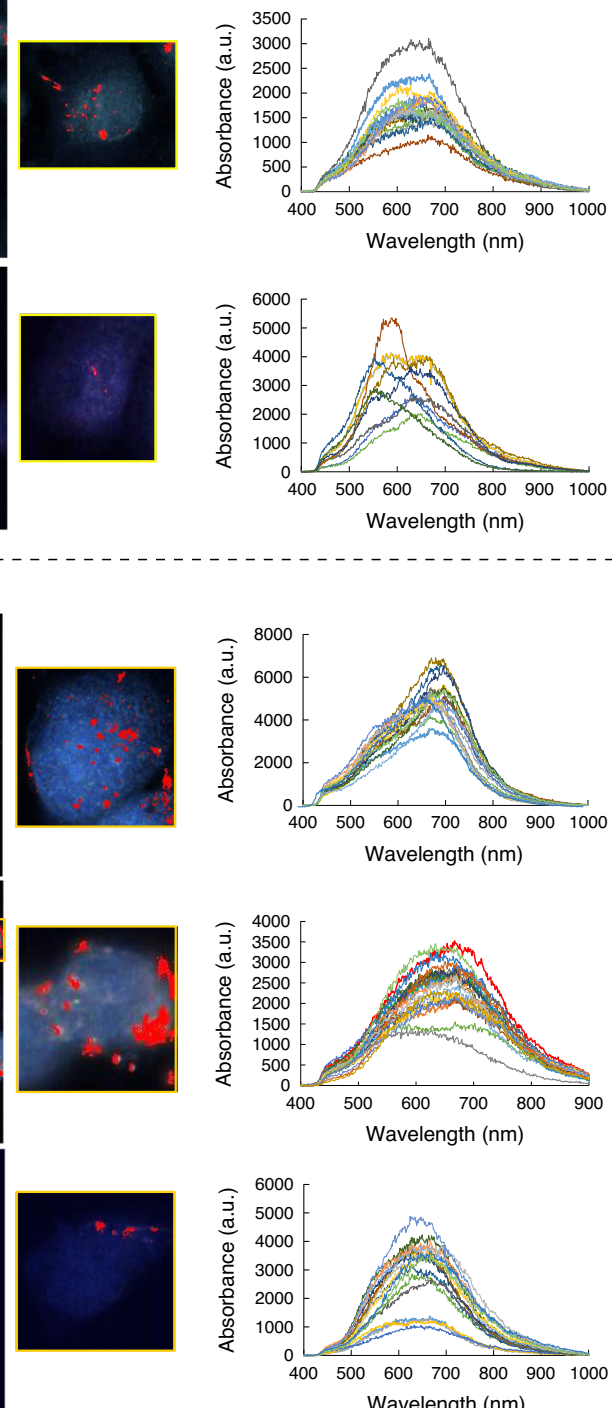

Reference SPLs for SAM
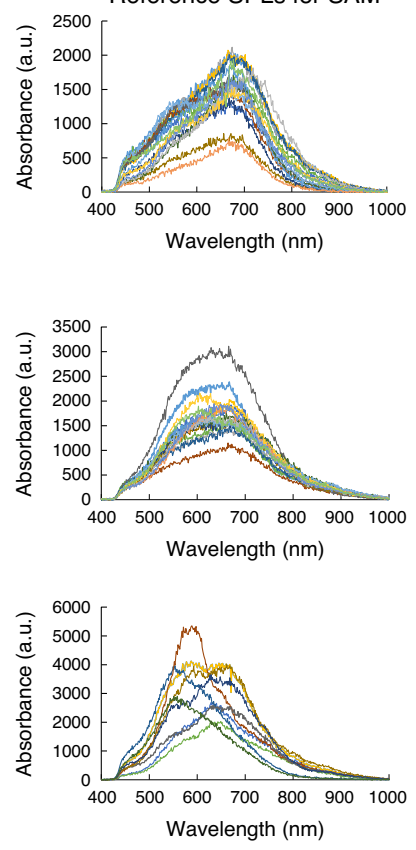

Fig. 5 Spectrally mapping of gold nanoparticles in cells using the spectral angle mapper algorithm. a Spectrally mapped gold particles in A549 cells with the SAM algorithm against the SPL (right panel) to localize particles in different regions of cells, $\mathbf{b}$ Spectrally mapped gold particles in HepG2 cells with the SAM algorithm against the SPL (right panel) to localize particles in different regions of HepG2 cells. Scale bar is $20 \mu m$

such as proteins and/or lipids, or changes in abiotic parameters, such as intracellular $\mathrm{pH}$. For this large shift, however, changes in the microenvironment seem to be more significant than interactions with biochemical components $^{42}$. Particles interact with different subcellular compartments, so the local environment surrounding particles is dramatically changed, altering the optical properties of their local regions. The effects of these changes can be reflected in the spectral responses. As shown in Fig. 6a, the LSPR for particles situated outside HepG2 cells is sharper, while for inside particles, it becomes broader, likely due to differences in the 


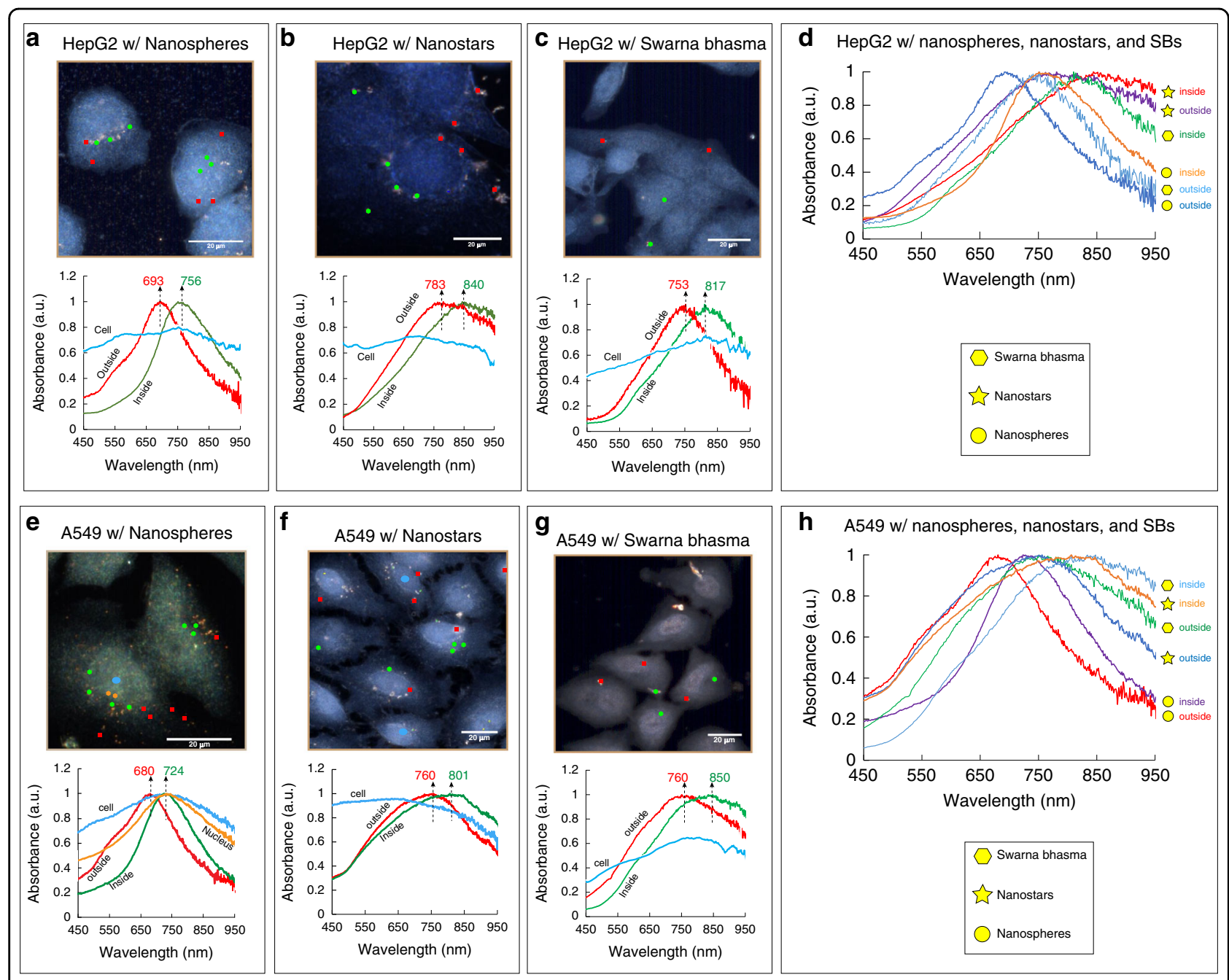

Fig. 6 Spectral responses of gold particles in different regions of both cell lines (outside or periphery and inside). a HepG2 cells with sphereshaped particles showing a plasmonic shift from $693 \mathrm{~nm}$ to $756 \mathrm{~nm}$ for outside and inside particles. b HepG2 cells with nanostars, showing a plasmonic shift from $783 \mathrm{~nm}$ to $840 \mathrm{~nm}$ for outside and inside particles. c HepG2 cells with SB particles and the plasmonic shift from $753 \mathrm{~nm}$ to $817 \mathrm{~nm}$. d Spectra of three different morphologies of particles in different regions of HepG2. e A549 cells with sphere-shaped particles showing a plasmonic shift from $680 \mathrm{~nm}$ to $724 \mathrm{~nm}$ for outside and inside particles. f A549 cells with nanostars, showing a plasmonic shift from $760 \mathrm{~nm}$ to $801 \mathrm{~nm}$. g A549 cells with SB particles and the plasmonic shift from $760 \mathrm{~nm}$ to $850 \mathrm{~nm}$. $\mathbf{h}$ Spectra of three different morphologies of particles in different regions of A549 cells. Scale bar is $20 \mu \mathrm{m}$

microenvironment. Spectra are also influenced by the size and aggregation of particles. The size of nanospheres is more consistent than the other two types, and their plasmonic shifts can be solely correlated to their intracellular interactions and environments. However, for SBs and nanostars, the effects of size and degree of aggregation should be taken into account in interpreting their plasmonic shifts. For nanostars, the bands shifted from $760-790 \mathrm{~nm}$ to $830-860 \mathrm{~nm}$ for particles localized at the periphery and inside HepG2 cells, respectively (Fig. 6b). SB particles are larger, and only a few smaller particles were inside the cells. Their bands shifted similar to those of nanospheres but were broader (Fig. 6c). Due to the irregular morphology and varying size of SBs, their LSPR changes may also be associated with their size and morphology.

A similar comparison was made for A549 cells with the three different types of particles to characterize their interactions with subcellular components. Since the nuclear region was clearer in these images, particles in the nucleus were also measured (orange). For gold nanospheres, the peak shifted to the right from $680 \mathrm{~nm}$ to $724 \mathrm{~nm}$ for particles outside/in the periphery of cells compared to particles in the cytosol (Fig. 6e). The LSPR band of particles in the nucleus was similar to that of particles in the cytosol but broader (full width at half maximum). The LSPR of smaller particles is sensitive than that of larger particles ${ }^{43}$ to the surrounding region/ 
Table 1 Effects of the surrounding medium and morphology of NPs on intracellular plasmonics

\begin{tabular}{lllll}
\hline Cell line & & Nanospheres & Nanostars & Swarna Bhasma \\
& & Spherical & Branched & Nonuniform \\
\hline A549 cells & Plasmonic shift $(\Delta \lambda)^{\mathrm{a}}$ & $\sim 44 \pm 9 \mathrm{~nm}$ & $\sim 41 \pm 7 \mathrm{~nm}$ & $\sim 90 \pm 14 \mathrm{~nm}$ \\
& Broadness change ${ }^{\mathrm{b}}$ & $+13.6 \%$ & $-8.9 \%$ & $+19.1 \%$ \\
& Q-factor (inside cells) & 4.55 & 2.68 & 4.45 \\
HepG2 Cells & Plasmonic shift & $\sim 57 \pm 8 \mathrm{~nm}$ & $+48 \pm 10 \mathrm{~nm}$ & $\sim 54 \pm 10 \mathrm{~nm}$ \\
& Broadness change & $+9.1 \%$ & $+19.6 \%$ & $+4.3 \%$ \\
& Q-factor & 4.72 & 2.46 & 3.82 \\
\hline
\end{tabular}

${ }^{\mathrm{a}} \Delta \mathrm{\lambda}=\lambda_{\text {inside }}-\lambda_{\text {outside }}$

${ }^{\mathrm{b}} \Delta w=100 \times \frac{w_{\text {inside cytoplasm }}-w_{\text {periphery of cells }}}{w^{w}}(w$ : full width at half maximum)

${ }^{c} Q=\frac{\lambda}{w}(\lambda$ : peak frequencen, $w$ : full width at half maximum)

surface, so they can reflect changes in their environment better. Therefore, the smaller size of nanospheres allows them to better sense their surrounding changes, and they appeared to be more sensitive to changes in their local environment. Another noticeable change in spectral responses was the peak broadness. The broadness was increased by $10-15 \%$ for nanospheres situated in the cells compared to nanospheres localized at the periphery of cells (Fig. 6e). The broadness change (\%) was determined by measuring the increase or decrease in the LSPR peak width (at half maximum) of NPs inside cells compared to the NPs outside or in the periphery of cells. In contrast to nanostars and SBs, a few nanospheres were also found in the nucleus of A549, and as shown in Fig. 6e, their plasmonic bands were shifted while they became broader compared to the NPs in the cytosol. In addition to the different interactions of NPs with subcellular compartments, the differences in dielectric constants could be another reason for the plasmonic shifts between particles situated in the periphery and particles in the cytoplasm or the nucleus. Some studies show that the cytoplasm of cells has dielectric constants ten times higher than that of the cell membrane ${ }^{41,44}$, and in another study ${ }^{45}$, the dielectric constant of the nucleus was found to be four times higher than that of the cytoplasm. Nanostars tend to aggregate, and their LSPR peaks were broader than those of nanospheres. Their peaks shifted from $760 \mathrm{~nm}$ to $801 \mathrm{~nm}$ for particles/aggregates outside cells compared to those inside cells (Fig. 6f). SBs are larger, and only a few were found inside cells where they randomly localized in the cytosol. The mean band for particles inside cells shifted from $760 \mathrm{~nm}$ (outside) to $850 \mathrm{~nm}$ (inside), and the peaks were broad, likely reflecting their nonuniform morphologies (Fig. 6g).

The plasmonic shifts for different cells and particles are reported in Table 1. The plasmonic shift takes place for all three types of particles between 41 and $90 \mathrm{~nm}$ depending on the type of cells, the morphology of particles, and their subcellular location. The data in Table 1 indicate a more consistent behavior of nanospheres in terms of peak broadness and the plasmonic shift, while two other particles exhibited different peak broadnesses in two different cell lines, likely due to the nonidentical shapes of SBs and the different aggregation levels of nanostars. The spectral profiles of nanostars revealed that they aggregated at the periphery and inside the cells. Therefore, nanostar plasmonic shifts from outside to inside cells can reflect both particleparticle interactions and particle-subcellular interactions. The results also indicated that the plasmonic shifts are stronger for SB particles whose size is larger compared to other particles. It seems that higher shifts in SBs originate mostly from the induced changes between particles rather than interactions with subcellular systems. Among these three particles, nanospheres are the best candidates to reveal information on the local environment due to their effective interactions with their surrounding medium. Any shifts in their bands can be correlated to the different subcellular regions of cells with better accuracy than two other particles. In addition, nanospheres offer higher quality factors (Qfactor) than the two other types of particles (Table 1). The quality factor was calculated as the ratio between the LSPR peak $(\mathrm{nm})$ and the width at half maximum $(\mathrm{nm})^{46}$. The quality factor shows the energy losses for the coherent motion of the electron and depends on the size, morphology, and surrounding medium of the NPs. For surrounding medium sensing, resonances with higher quality factors are desirable (narrow widths) ${ }^{47-49}$. Moreover, our results showed that SB particles and nanostars have limitations in reaching different subcellular regions of cells. However, due to their smaller sizes, nanospheres were found in different subcellular regions in cells, enabling their use for subcellular diagnosis through intracellular plasmonics.

\section{Particle distribution in cells}

After entering cells, particles were localized in three different regions: at the periphery or distant from the 


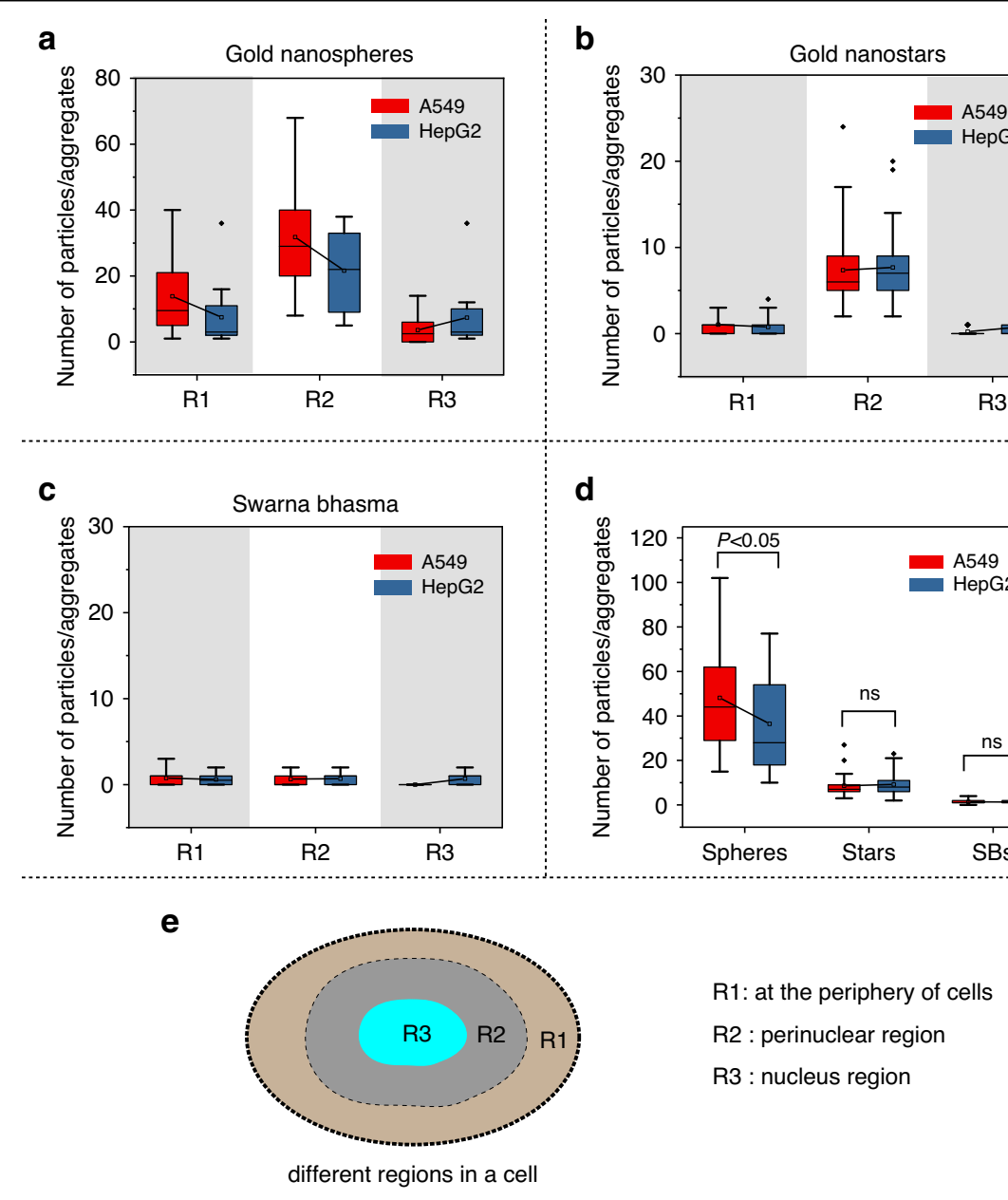

Fig. 7 Gold nanoparticles depending on their nanomorphology and size, are distributed differently in different regions of cells. a Nanosphere particle distribution in different regions of cells for both cell lines, $\mathbf{b}$ gold nanostar distribution in different regions of cells for both cell lines, c SB particle distributions in cells for both cell lines, $\mathbf{d}$ the total number of particles in two cell lines, e different regions in cells (R1: far from the nucleus and at the cell periphery, R2: perinuclear region, and R3: nucleus)

nucleus, in the perinuclear region, and inside the nucleus. Discrete nanospheres could be identified in different parts of cells, while nanostars aggregated either inside cells or at their periphery. SB particles are much larger, the size of their aggregates might reach one micron, and their internalization level was not high. To estimate the number of particles in cells, the occupied area of particles in cells was measured, and by considering the average size of particles, they were counted in cells. At least 150 cells were considered for each type of particle, and their distributions were quantified in three different (Fig. 7e) regions and plotted in Fig. 7.

The average number of nanospheres per cell was higher for both cell lines than the two other types of particles, and A549 cells had almost two times more nanospheres. Note that A549 cells are larger in comparison to HepG2 cells, and they could accommodate more particles. There was no significant difference between the particle numbers of the two other particles in the two cell lines. In contrast to nanostars and nanospheres, the uptake of SB particles in cells was very low, and their distributions in different regions were random. It is evident that particles (nanospheres and nanostars) were mostly localized in cells and at the periphery of the nucleus (Fig. 7a, b). This pattern can be seen in both cell lines. SB particles were few (with smaller size) in cells and did not follow a specific distribution pattern in cells.

\section{Conclusions and outlook}

In NP-based cancer therapy, precise subcellular detection/distribution of NPs is crucial to designing effective nanomedicines. Although the detection of NPs in biological environments has been accomplished using electron microscopy, newer, cheaper, more advanced, and faster methodologies provide opportunities. The highly sensitive optical properties of gold NPs have 
provided a novel and noninvasive platform to develop LSPR-based methods to diagnose NPs at subcellular levels. In this work, this idea was verified using three different gold particles with different morphologies. We showed how hyperspectral imaging and spectral measurements at the nanoscale enable us to approximate their localization in different subcellular regions and understand their interactions with subcellular compartments. Intracellular diagnosis with hyperspectral imaging allows not only confirmation of the presence of particles in cells but also nanoscale spectral measurements to provide information on their surrounding medium. Inside the cell, there are various microenvironments, and gold particles localized in these regions exhibit different plasmonic shifts depending on the optical properties of their surrounding regions, as well as their physicochemical properties. Our results revealed that particles could sense their intracellular environments. We examined particle responses at different subcellular regions, at the periphery of cells, at the perinuclear and, inside the nucleus. Our measurements showed that particles, depending on their interactions with their local environments, have different intracellular plasmonic responses. During these interactions, the surrounding medium of particles is changed, affecting the LSPR properties of NPs. Stability and consistency in both morphology and size of particles are two important factors for subcellular detection. Due to the inconsistency and irregular shape of SBs and the strong tendency of nanostars to aggregate, the plasmonic shift in their responses cannot be purely correlated to modifications in their surrounding mediums and how they interact with subcellular compartments. The effects of particle-particle interactions and the morphology of particles could also be reflected by plasmonic shifts. Among the three particles, nanospheres were distributed more uniformly within cells and appeared to reflect the effects of their surrounding environments better, as they showed a more consistent behavior in cells. Furthermore, the distribution of particles showed that nanospheres are capable of navigating to different subcellular regions in cells, while the two other particles had limited access to different subcellular regions. Designing proper NPs with specific physicochemical properties will play a critical role in subcellular diagnosis with hyperspectral imaging. For future works, the unique spectral signature of gold NPs and plasmonic shifts of NPs can be used to create a comprehensive library for different subcellular regions/organelles of cells for more accurate subcellular sensing. This is feasible when the HSI-based subcellular diagnostic technique is simultaneously used with highresolution microscopic techniques to visualize subcellular regions/organelles with more details. This library can be used to detect NPs at subcellular levels, accurately predict their localizations in cells, and study how they interact with their subcellular compartments.

\section{Acknowledgements}

Natural Sciences and Engineering Research Council of Canada (NSERC),

Concordia Research Chair (CURC) and Fonds Québécois de la Recherche sur la Nature et les Technologies (FRQNT) grants to M.P., NSERC grant to A.P., and doctoral FRQNT to A.S.K. are acknowledged.

\section{Author contributions}

M.P. and A.S.K. conceived the study. A.S.K. performed the experiments, analysis, and drafted the manuscript. A.P. provided the support for biological experiments. All authors reviewed the manuscript.

\section{Conflict of interest}

The authors declare that they have no conflict of interest.

Received: 29 December 2019 Revised: 17 September 2020 Accepted: 24 September 2020

Published online: 14 December 2020

\section{References}

1. Villaverde, G. \& Baeza, A. Targeting strategies for improving the efficacy of nanomedicine in oncology. Beilstein J. Nanotechnol. 10, 168-181 (2019).

2. Shittu, K. O., Bankole, M. T., Abdulkareem, A. S., Abubakre, O. K. \& Ubaka, A. U. Application of gold nanoparticles for improved drug efficiency. Adv. Nat. Sci. Nanosci. Nanotechnol. 8, 035014 (2017).

3. Marrow, B., Secreted, S. \& Protect, C. Insight into nanoparticle cellular uptake and intracellular targeting. J. Control Release 71, 3831-3840 (2014).

4. Sanna, V., Pala, N. \& Sechi, M. Targeted therapy using nanotechnology: focus on cancer. Int. J. Nanomed. 9, 467-483 (2014).

5. Ostrowski, A. et al. Overview about the localization of nanoparticles in tissue and cellular context by different imaging techniques. Beilstein J. Nanotechnol. 6. 263-280 (2015).

6. D'Acunto, M. Detection of intracellular gold nanoparticles: an overview. Materials 11, 882 (2018).

7. Pancani, E. et al. High-resolution label-free detection of biocompatible polymeric nanoparticles in cells. Part. Part. Syst. Charact. 35, 1700457 (2018).

8. Drasler, B., Vanhecke, D., Rodriguez-Lorenzo, L., Petri-Fink, A. \& RothenRutishauser, B. Quantifying nanoparticle cellular uptake: Which method is best? Nanomedicine 12, 1095-1099 (2017).

9. Shapero, K. et al. Time and space resolved uptake study of silica nanoparticles by human cells. Mol. Biosyst. 7, 371-378 (2011).

10. Efeoglu, E., Keating, M., Mclntyre, J., Casey, A. \& Byrne, H. J. Determination of nanoparticle localisation within subcellular organelles in vitro using Raman spectroscopy. Anal. Methods 7, 10000-10017 (2015).

11. Chen, S. et al. Direct observation of nanoparticles within cells at subcellular levels by super-resolution fluorescence imaging. Anal. Chem. 91, 5747-5752 (2019).

12. Sohrabi Kashani, A., Badilescu, S., Piekny, A. \& Packirisamy, M. Differing affinities of gold nanostars and nanospheres toward HeLa and HepG2 cells: implications for cancer therapy. ACS Appl. Nano Mater. 3, 4114-4126 (2020).

13. Taylor, J., Huefner, A., Li, L., Wingfield, J. \& Mahajan, S. Nanoparticles and intracellular applications of surface-enhanced Raman spectroscopy. Analyst 141, 5037-5055 (2016).

14. Kashani, A. S., Badilescu, S., Piekny, A. \& Packirisamy, M. Bio-nano-interaction in treatment and management of cancer. J. Electrochem. Soc. 166, B3007-B3011 (2019).

15. Zhu, J. et al. Surface-enhanced Raman spectroscopy investigation on human breast cancer cells. Chem. Cent. J. 7, 37 (2013).

16. Schie, I. W. \& Huser, T. Methods and applications of Raman microspectroscopy to single-cell analysis. Appl. Spectrosc. 67, 813-828 (2013).

17. Hermida-Ramon, J. M., Guerrini, L. \& Alvarez-Puebla, R. A. Analysis of the SERS spectrum by theoretical methodology: Evaluating a classical dipole model and the detuning of the excitation frequency. J. Phys. Chem. A 117, 4584-4590 (2013). 
18. Kuruvinashetti, K. et al. Intracellular localized surface plasmonic sensing for subcellular diagnosis. Plasmonics 13, 1639-1648 (2018).

19. Crane, N. J., Huffman, S. W., Gage, F. A., Levin, I. W. \& Elster, E. A. Evidence of a heterogeneous tissue oxygenation: renal ischemia/reperfusion injury in a large animal. J. Biomed. Opt. 18, 035001-035007 (2003).

20. Mehta, N., Shaik, S., Devireddy, R. \& Gartia, M. R. Single-cell analysis using hyperspectral imaging modalities. J. Biomech. Eng. 140, 020802 (2018).

21. Juan-Colás, J., Hitchcock, I. S., Coles, M., Johnson, S. \& Krauss, T. F. Quantifying single-cell secretion in real time using resonant hyperspectral imaging. Proc. Natl Acad. Sci. USA 115, 13204-13209 (2018).

22. Lu, G. \& Fei, B. Medical hyperspectral imaging: a review. J. Biomed. Opt. 19, 010901 (2014)

23. Lu, G. et al. Spectral-spatial classification for noninvasive cancer detection using hyperspectral imaging. J. Biomed. Opt. 19, 106004 (2014).

24. Mahadevan-Jansen, A. Raman spectroscopy for the detection of cancers and precancers. J. Biomed. Opt. 1, 31 (1996).

25. Smith, R., Wright, K. L. \& Ashton, L. Raman spectroscopy: an evolving technique for live cell studies. Analyst 141, 3590-3600 (2016).

26. Singh, M. R., Chandra Sekhar, M., Balakrishnan, S. \& Masood, S. Medical applications of hybrids made from quantum emitter and metallic nanoshell. J. Appl. Phys. 122, 034306 (2017).

27. Singh, M. R., Guo, J., José, J. M. \& De Hoyos Martinez, J. E. Control of fluorescence in quantum emitter and metallic nanoshell hybrids for medical applications. J. Appl. Phys. 121, 094303 (2017).

28. Singh, M. R., Guo, J., Fanizza, E. \& Dubey, M. Anomalous photoluminescence quenching in metallic nanohybrids. J. Phys. Chem. C. 123, 10013-10020 (2019).

29. Kashani, A. S. et al. Enhanced internalization of indian ayurvedic swarna bhasma (Gold Nanopowder) for effective interaction with human cells. J. Nanosci. Nanotechnol. 18, 6791-6798 (2018).

30. Beaudet, D. et al. Comparative study on cellular entry of incinerated ancient gold particles (Swarna Bhasma) and chemically synthesized gold particles. Sci. Rep. 7, 1-11 (2017).

31. Turkevich, J., Stevenson, P. C. \& Hillier, J. A study of the nucleation and growth processes in the synthesis of colloidal gold. Discuss. Faraday Soc. 11, 55-75 (1951).

32. SoRelle, E. D. et al. A hyperspectral method to assay the microphysiological fates of nanomaterials in histological samples. Elife 5, e16352 (2016).

33. Singh, M. R. \& Black, K. Anomalous dipole-dipole interaction in an ensemble of quantum emitters and metallic nanoparticle hybrids. J. Phys. Chem. C. 122 26584-26591 (2018)
34. Keh, H. J. Diffusiophoresis of charged particles and diffusioosmosis of electrolyte solutions. Curr. Opin. Colloid Interface Sci. 24, 13-22 (2016).

35. Karmakar, S. Particle size distribution and zeta potential based on dynamic light scattering: techniques to characterise stability and surface distribution of charged colloids. Recent Trends in Materials Physics and Chemistry (Studium Press(India)Pvt Ltd, 2019). pp 117-159.

36. Xiao, L. \& Yeung, E. S. Optical imaging of individual plasmonic nanoparticles in biological samples. Annu. Rev. Anal. Chem. 7, 89-111 (2014).

37. Fairbairn, N., Christofidou, A., Kanaras, A. G., Newman, T. A. \& Muskens, O. L. Hyperspectral darkfield microscopy of single hollow gold nanoparticles for biomedical applications. Phys. Chem. Chem. Phys. 15, 4163-4168 (2013).

38. Kumar, S., Harrison, N., Richards-Kortum, R. \& Sokolov, K. Plasmonic nanosensors for imaging intracellular biomarkers in live cells. Nano Lett. 7, 1338-1343 (2007).

39. Willets, K. A. \& Van Duyne, R. P. Localized surface plasmon resonance spectroscopy and sensing. Annu. Rev. Phys. Chem. 58, 267-297 (2007).

40. Dmitriev, A. Nanoplasmonic Sensors. Nanoplasmonic Sensors (Springer New York, 2012).

41. Adams, T. N. G., Turner, P. A., Janorkar, A. V., Zhao, F. \& Minerick, A. R. Characterizing the dielectric properties of human mesenchymal stem cells and the effects of charged elastin-like polypeptide copolymer treatment. Biomicrofluidics 8, 1-14 (2014).

42. Messersmith, R. E., Nusz, G. J. \& Reed, S. M. Using the localized surface plasmon resonance of gold nanoparticles to monitor lipid membrane assembly and protein binding. J. Phys. Chem. C. 117, 26725-26733 (2013).

43. Guo, L. et al. Strategies for enhancing the sensitivity of plasmonic nanosensors. Nano Today 10, 213-239 (2015).

44. Theillet, F. X. et al. Physicochemical properties of cells and their effects on intrinsically disordered proteins (IDPs). Chem. Rev. 114, 6661-6714 (2014).

45. Sasmal, D. K., Ghosh, S., Das, A. K. \& Bhattacharyya, K. Solvation dynamics of biological water in a single live cell under a confocal microscope. Langmuir $\mathbf{2 9}$, 2289-2298 (2013).

46. Lilley, G., Messner, M. \& Unterrainer, K. Improving the quality factor of the localized surface plasmon resonance. Opt. Mater. Express 5, 2112 (2015).

47. Johns, P., Beane, G., Yu, K. \& Hartland, G. V. Dynamics of surface plasmon polaritons in metal nanowires. J. Phys. Chem. C. 121, 5445-5459 (2017).

48. Hartland, G. V. Optical studies of dynamics in noble metal nanostructures. Chem. Rev. 111, 3858-3887 (2011).

49. Hu, M. et al. Dark-field microscopy studies of single metal nanoparticles: understanding the factors that influence the linewidth of the localized surface plasmon resonance. J. Mater. Chem. 18, 1949-1960 (2008). 